\title{
Erzählen im Reallabor. Ein Beitrag zur konzeptionellen Ausgestaltung partizipativer Methoden der gemeinsamen Wissensproduktion durch Erzählräume im Reallabor
}

\author{
Hanna Seydel (, Katrin Gliemann, Sandra Stark, Bianca Herlo \\ Eingegangen: 1. August 2020 - Angenommen: 14. April 2021 - Online veröffentlicht: 5. Mai 2021
}

\begin{abstract}
Zusammenfassung
Die transdisziplinäre Schnittstelle zwischen Wissenschaft und Gesellschaft wird im Reallaboransatz als Potenzial für die Erzeugung von Transformationswissen angesehen. Gleichzeitig gibt es offene Fragen bei der methodischen Umsetzung, denen in diesem Beitrag mit Erkenntnissen über experimentelle Formate des Erzählens begegnet wird. Am Beispiel des Forschungsprojekts INTERPART wird den Fragen nachgegangen, wie sich interkulturelle Räume der Partizipation in der Stadtentwicklung erweitern lassen und welche institutionellen Veränderungsprozesse dafür erforderlich sind. Zusätzlich involviert sind zwei wissenschaftliche Disziplinen - Raumplanung und Designforschung - und Praxisfelder. Eine zentrale Erkenntnis des Projekts ist, wie bewusst gestaltete Erzählsituationen die Ko-Produktion von transformativem Wissen fördern, indem sie Partizipation in der Forschung begünstigen und Stadtnutzer/-innen als Expertinnen und Experten
\end{abstract}

$\triangle$ Hanna Seydel, Fakultät Raumplanung, Technische Universität Dortmund, August-Schmidt-Straße 10, 44227 Dortmund, Deutschland

hanna.seydel@tu-dortmund.de

Dr. Katrin Gliemann, Fakultät Raumplanung, Technische Universität Dortmund, August-Schmidt-Straße 6, 44227 Dortmund, Deutschland

katrin.gliemann@tu-dortmund.de

Sandra Stark, Design Research Lab, Universität der Künste Berlin, Einsteinufer 43, 10587 Berlin, Deutschland

s.stark@udk-berlin.de

Dr. Bianca Herlo, Design Research Lab, Universität der Künste Berlin, Einsteinufer 43, 10587 Berlin, Deutschland

bianca.herlo@udk-berlin.de

(c) (1) ( 2021 Seydel; licensee oekom verlag. This Open Access article is published under a Creative Commons Attribution 4.0 International License. ihres Alltags in die Ko-Forschung einbinden. Die Ergebnisse stellen somit einen Beitrag zum aktuellen Diskurs um partizipative Forschung und Ko-Produktion von Wissen im Kontext methodischer Ausdifferenzierung von Reallaboransätzen dar.

Schlüsselwörter: Transdisziplinarität - Ko-Produktion Narrative Forschung - Designforschung - Partizipation Stadtentwicklung - Berlin-Moabit - Wiesbaden-Biebrich

Storytelling in the real-world laboratory. A contribution to the conceptual design of participatory methods for the co-production of knowledge through storytelling in the realworld laboratory

\footnotetext{
Abstract

The transdisciplinary intersection between science and society in the real-world-laboratory approach is seen as a potential for generating transformational knowledge. At the same time, there are open questions in the methodological implementation, which are addressed in this paper by insights on experimental formats of storytelling. Using the example of the research project INTERPART, this paper explores the questions how intercultural spaces of participation in urban development can be expanded and what changes in institutional processes are required to achieve this. The paper involves two academic disciplines - spatial planning and design research and fields of practice. Central findings include how deliberately designed narrative situations promote the co-production of transformative knowledge by encouraging participation in research and engaging citizens as experts of everyday life in co-research. The paper thus contributes to the current dis-
} 
course on participatory research and co-production of knowledge in the context of methodological differentiation of realworld laboratory approaches.

Keywords: Transdisciplinarity - Co-production - Narrative research - Design research - Participation - Urban development $\mathbf{- B}$ Berlin-Moabit $\mathbf{-}$ Wiesbaden-Biebrich

\section{Einführung}

Ein wesentliches Merkmal des transdisziplinär angelegten Reallaboransatzes ist die Verschränkung von Partizipation und Forschung. Im Reallabor umfasst Partizipation ,die Teilhabe und Teilnahme [von lokalen Akteuren] an Projektarbeit, Forschung und gesellschaftlichen Gestaltungsprozessen [und] spielt für das Verständnis der transdisziplinären Forschung eine tragende Rolle" (Parodi/Beecroft/ Albiez et al. 2016: 13). Dass Reallabore Anforderungen von Forschung und Partizipation simultan gerecht werden müssen, ist mit besonderen Schwierigkeiten verbunden, denn es geht darum, ,gleichermaßen reale Transformationsprozesse anzustoßen (Praxisziele) und - soweit möglich - verallgemeinerbares Wissen über Transformation hervorzubringen (Forschungsziele), wie auch darum, Lernen zu ermöglichen (Bildungsziele)“ (Eckart/Ley/Häußler et al. 2018: 105).

In diesem Beitrag befassen wir uns mit partizipativen, gestalteten Erzählsituationen in Reallaboren, die den Erfahrungen und dem (Alltags-)Wissen der Stadtnutzer/-innen in Form von biographisch eingebetteten Erzählungen als alltagskommunikative Praktiken (Bruner 2004: 691 ff.) Raum geben und sie aufgreifen (im Folgenden Formate des Erzählens, Erzählräume und Erzählsituationen). Wir argumentieren, dass die Erzählräume eine Möglichkeit darstellen, um der Herausforderung der Gleichzeitigkeit von Partizipation in der Stadtentwicklung als Thema und Partizipation an der Forschung als Vorgehen zu begegnen.

Erzählungen im Sinne des Reallaboransatzes besitzen unseres Erachtens transformatives Potenzial, indem sie Menschen ermöglichen, ihr Erfahrungswissen über das Leben in der Stadt in der eigenen Sprache bzw. Ausdrucksweise einzubringen und im Austausch mit Wissenschaftlerinnen und Wissenschaftlern die Relevanz ihrer Alltagserfahrungen und ihre Handlungsmöglichkeiten verstehen lernen. Das Teilen und Sichtbarmachen von unterschiedlichen Perspektiven, Interessen, Wertvorstellungen und Wissensbeständen kann bei Wissenschaftlerinnen/Wissenschaftlern, Stadtnutzerinnen/-nutzern und Verwaltungsmitarbeiterinnen/-mitarbeitern gleichermaßen Lernprozesse anstoßen und damit das Verhältnis von Wissenschaft zu Gesellschaft transformieren. Der bewusste Umgang mit Erzählräumen kann so- mit zur transformativen Wissensproduktion über Stadt beitragen.

Vor diesem Hintergrund widmen wir uns der Frage, wie Ko-Produktion als Teil der Ko-Forschung mithilfe von spezifischen narrativen Partizipationsmethoden funktioniert: Welche Erzählsituationen können genutzt werden? Wie müssen sie gestaltet werden, um erkenntnisgewinnend und transformativ zu wirken? Wir beziehen uns auf Erkenntnisse aus dem Forschungsprojekt INTERPART ${ }^{1}$, das den Reallaboransatz (vgl. Schneidewind 2014; Gerhard/Marquardt 2017; Schäpke/Stelzer/Bergmann et al. 2017) nutzt, um der Frage nachzugehen, wie sich interkulturelle Räume der Partizipation und des Dialogs in der Stadtentwicklung, unter anderem mittels digitaler Dienste, erweitern lassen und welche institutionellen Veränderungsprozesse dafür erforderlich sind.

INTERPART hat zum Ziel, Beteiligungsformate in der Stadtentwicklung interkulturell weiterzuentwickeln, um urbane Vielfalt umfassender als bisher in Beteiligungsprozessen abzubilden: Welche institutionellen Ressourcen, Kompetenzen und Kapazitäten sind dafür erforderlich? Welche Themen und Formate sind geeignet und welche Potenziale ergeben sich für Stadtentwicklungsprozesse und städtische Teilhabe? Anders als in herkömmlichen Beteiligungsprozessen ist die Aktivität von INTERPART allerdings nicht an einem konkreten Planungsvorhaben orientiert, sondern agiert unabhängig von solch einem ortsspezifischen Anlass. Das Projekt verfolgt einen breiten methodischen Ansatz, die Erzählformate als Fokus dieses Beitrags sind nur ein Baustein. In dem transdisziplinären Projekt arbeiten sechs Partner zusammen, wobei sich dieser Beitrag vor allem auf die Kooperation der Disziplinen Raumplanung und Designforschung bei der Konzeption von Erzählsituationen bezieht.

Im nachfolgenden Kapitel 2 gehen wir auf den transdisziplinären Ansatz des Reallabors und die Bedeutung der Ko-Forschung ein und spezifizieren diese im Zuge einer Verknüpfung der Perspektiven von Raum- und Designforschung. Es folgt in Kapitel 3 eine Auseinandersetzung mit Erzähltheorien und dem Mehrwert von Erzählung als methodischem Zugang im Bereich der Raumplanung, um Erfahrungswissen hervorzuholen. Nach einem Zwischenfazit (Kapitel 4) berichten wir in Kapitel 5 von praktischen Erfahrungen mit Erzählsituationen und narrativen Strategien

\footnotetext{
1 https:// www.interpart.org (15.03.2021). Partner: Technische Universität Dortmund, Fakultät Raumplanung, Fachgebiete Stadtund Regionalsoziologie sowie International Planning Studies; Design Research Lab der Universität der Künste Berlin; Agentur für crossmediale Bürgerbeteiligung Zebralog; UP19 Stadtforschung + Beratung $\mathrm{GmbH}$; Landeshauptstadt Wiesbaden (Stabstelle Wiesbadener Identität. Engagement. Bürgerbeteiligung); Land Berlin (Senatsverwaltung für Stadtentwicklung und Wohnen).
} 
im Reallabor des Forschungsprojekts INTERPART. In Kapitel 6 fassen wir zentrale Erkenntnisse zusammen, beleuchten die Grenzen der Formate und skizzieren die Herausforderungen zukünftiger Reallaborprojekte. Zum Abschluss in Kapitel 7 setzen wir unsere Erfahrungen in einen größeren Kontext.

\section{Rezeption des Reallabors in Raum- und Designforschung}

Vor dem Hintergrund komplexer realweltlicher Probleme lässt sich in den vergangenen zwei Jahrzehnten ein verändertes Verhältnis von Wissenschaft und Gesellschaft beobachten, diskutiert unter dem Begriff ,Transformative Wissenschaft". In diesem Beitrag beziehen wir transformative Wissenschaft vor allem auf die Ko-Produktion von Wissen mit denen, die die Stadt auch nutzen. Die Herausforderung besteht darin, einen kommunikativen Raum zu schaffen, in dem durch partizipative Forschung der Prozess der kollaborativen Wissensproduktion gelingt. Dafür hat sich das Zusammenspiel unterschiedlicher Kompetenzen in der Kooperation von Raumplanung und Designforschung als sehr fruchtbar erwiesen.

Die Designforschung bezieht sich in den letzten Jahren wieder stark auf partizipative und kollaborative Möglichkeiten der Wissensproduktion in der nachhaltigen Produktund Prozessgestaltung (Sanders 2006; Mareis 2010; Mareis 2011; Sanders 2013; Joost/Unteidig 2015). Participatory Design und Social Design als Arbeitsbereiche innerhalb der Designdisziplin markieren eine Wende, derzufolge es immer weniger um die von realweltlichen Bedarfen entkoppelte Gestaltung von Artefakten geht und mehr um die frühe Einbindung von Nutzerinnen und Nutzern in Entwurfsprozesse als ,Expertinnen und Experten ihres Alltags“ (Hörning 2001; Björgvinsson/Ehn/Hillgren 2010).

In der raumbezogenen Forschung und Planung lässt sich das große Interesse an Reallaboren zunächst durch die Nähe der Planung zum Nachhaltigkeitsdiskurs erklären. Weitere Parallelen liegen darin, dass jeweils lokalspezifische Situationen unter Hinzuziehen von Vor-Ort-Expertinnen und -Experten verstanden werden sollen oder in Bezug auf Ansätze des Empowerments und der Partizipation (Kanning 2018: $7 \mathrm{f}$; Hahne 2019). Allerdings kann ein vorschneller Verweis auf den Reallabor-nahen Erfahrungsschatz der räumlichen Planung in die Irre führen, da $B e$-forschen, $B e$-teiligen und $B e$-planen auf dem Präfix ,be' aufbauen und damit weiterhin ein hierarchisches Verständnis implizieren, bei dem $b e-$ troffene Akteure gehört werden, aber einen Objektstatus beibehalten. Der Perspektivwechsel vom Be-Forschen zum KoForschen - hier verstanden als Sammelbegriff für die im Reallabor häufig verwendeten Bezeichnungen Ko-Design, Ko-
Produktion und Ko-Evaluation (Borner/Kraft 2018: 1, 12f.; Rose/Wanner/Hilger 2019: 6 ff.) - erweist sich allerdings als Herausforderung im Reallabor. Diese Herausforderung wird noch verschärft, wenn es sich um wissenschaftliche Fragen zu Partizipation an Planungsprozessen handelt.

Machtungleichgewichte ergeben sich in Reallaboren bereits aufgrund der Ausgangssituation: Insbesondere zivilgesellschaftliche Akteure sind (bestenfalls) mit konventioneller Partizipation vertraut, aber in aller Regel forschungsunerfahren, woraus schnell ein hierarchisches, mit Definitions- und Entscheidungsmacht verknüpftes Gefälle statt der geforderten Zusammenarbeit ,,auf Augenhöhe“ (Borner/ Kraft 2018: 16) entstehen kann. In der Planungspraxis werden aus Stadtnutzer/-innen ,zu Beteiligende“ in einem meist top-down organisierten Prozess. In Reallaboren nehmen sie neue Rollen ein, dies bedingt Herausforderungen für Wissenschaft und Bürgerinnen/Bürger und erfordert Aushandlungsprozesse. Zudem ist den ,zu Beteiligenden“ der Mehrwert der Teilnahme an Reallaboren schwer zu vermitteln, dies kann selektiv auf die Zusammensetzung der Teilnahmegruppen wirken.

Ein weiteres, für unser Thema besonders relevantes Hindernis resultiert daraus, dass selbst bei vorhandenem Interesse von Stadtnutzerinnen und -nutzern am Reallabor häufig die gemeinsame Sprachebene fehlt. Von Unger (2014: 85 ) konstatiert hinsichtlich partizipativer Forschung, die Bezugssysteme der beteiligten Gruppen verfügten über ,eine jeweils eigene Funktionsweise und Logik, und die Personen sprechen häufig eine unterschiedliche Sprache“. Sprache beziehen wir hier nicht (unbedingt) auf unterschiedliche Muttersprachen, sondern zunächst auf die Alltagssprache, sofern sie sich von planerischer Fachsprache unterscheidet.

Unsere These ist, dass gestaltete Erzählsituationen vielversprechende Anknüpfungspunkte an viele der beschriebenen Herausforderungen im Reallabor bieten. Durch das Erzählen entstehen Situationen, in denen die Stadtnutzer/-innen persönliche, biographisch geprägte Erfahrungen austauschen können. Im Unterschied zur klassischen Erhebung entsteht hier in der Interaktion sowohl mit den zuhörenden Wissenschaftlerinnen und Wissenschaftlern als auch der Teilnehmenden untereinander eine gegenseitige Bezugnahme, in der implizites Wissen (vgl. Polanyi 1985) explizit gemacht und ein geteiltes Wissen generiert wird. Dieses vielschichtige Wissen kann für den Forschungsprozess damit erschlossen werden. Besonders bei sensiblen Themenfeldern wie Diskriminierung oder Exklusion lassen sich durch Zuhören und Austausch von Erfahrungen schwierige Aspekte vielschichtig abrufen.

Unser Raumverständnis in diesem Beitrag bezieht sich auf die Räume der bzw. in den Vor-Ort-Interventionen dort, wo die Erzählenden leben, und in dem Raum, von dem die Erzählungen handeln. Raum wird dabei so verstan- 
den, dass soziale Beziehungen ihn formen (Massey 2005), wir legen also ein relationales Raumverständnis zugrunde. Das heißt, dass Raum immer ein Produkt von Wechselbeziehungen ist (Massey 2005). Wenn wir von Erzählräumen sprechen, dann spielt auch das Raumverständnis des New Materialism eine Rolle, in dem das Entstehen und Sein von Räumen als ein Prozess der Aushandlung verstanden wird, indem individuelle und kollektive Geschichten, Materialitäten, Machtkonstellationen, gleichzeitige und vorausgegangene Ereignisse Räume produzieren (Landolt 2010: 240).

Erzählräume zur Wissensproduktion werden bisher kaum in Reallaboren explizit genutzt. Zwar initiieren viele planungsbezogene Reallabore Dialogprozesse zwischen Stadtbevölkerung und Wissenschaft oder Verwaltung, aber dabei stehen nicht die Erzählungen als Austausch von Erfahrungen und Ko-Produktion von Wissen im Vordergrund. ${ }^{2}$ In der partizipativen Designforschung wird mit Erzählen als methodischem Zugriff, um Alltagswissen der Teilnehmenden und biographische Erfahrungen möglichst früh in den Entwurfsprozess einzubinden, bereits seit Längerem experimentiert (Unteidig/Domínguez Cobreros/CalderonLüning et al. 2017; Rössig/Herlo/Moormann et al. 2018). Basierend auf dem Ansatz des Social Living Lab, der speziell auf dem skandinavischen Modell des Living Lab aufbaut, ist insbesondere der experimentelle Charakter in spezifischen lokalen Kontexten relevant, um die strikten Grenzen zwischen akademischem und nichtakademischem Expertentum aufzulösen und Ergebnisoffenheit und Experiment zuzulassen (Dezuanni/Foth/Mallan et al. 2018: 9). Alltagspraktisches Erzählen als Vorstufe des Ko-Designs wird dafür eingesetzt, eine Vertrauensbasis zu schaffen und das community building für die Teilnehmenden am partizipativen Forschungs- und Entwicklungsprozess zu unterstützen. Allerdings wird im Bereich Social Living Lab der Zugriff als wissenschaftliche Methode noch nicht ausführlich bzw. systematisch ausgewertet. Spätestens hier wird der Mehrwert der interdisziplinären Zusammenarbeit deutlich: Die Erfahrungen mit narrativen Formaten in urbanen Kontexten und die gestalterischen Kompetenzen aus der Designforschung auf der einen und die Systematisierungs- und Auswertungskompetenzen der Raumforschung auf der anderen Seite ergänzen sich hier auf ideale Weise.

\footnotetext{
2 Grundlage für diese Aussage ist die Untersuchung von 50 Webpräsenzen von Reallaboren mit raumplanerisch orientierten Themen. Als Einschränkung ist jedoch zu nennen, dass es teilweise keine Detailangaben zu den Methoden gibt, da sich die Webseiten oft an die Zivilgesellschaft richten.
}

\section{Erzähltheorien im Überblick}

Wir unterscheiden drei Bereiche, von denen aus wir uns das Verständnis von Erzählen und Erzählungen erarbeiten: die Funktionen des Erzählens, Erzählungen als Produkt sowie die Gestaltung von Erzählräumen (vgl. Kapitel 5).

Die Ausführungen von Martínez (2011a; 2017) dienen uns als Ausgangspunkt im weiten Feld der Erzählforschung. Die Funktionen des Erzählens ergeben sich nach Martínez (2011b: 8) dadurch, dass Erzählen als Sprachhandlung unterschiedliche Funktionen beinhaltet: „Man kann erzählend informieren, unterhalten oder belehren, moralisch unterweisen, geistlich stärken oder politisch indoktrinieren, Erzählgemeinschaften bilden, individuelle oder kollektive Identitäten stiften usw. “ Eine Erzählung ist nicht nur eine persönliche Geschichte, sondern transportiert Informationen über die Sicht der Erzählenden auf ihre jeweilige Umwelt, z. B. Nachbarschaft und Quartier. Erzählungen sind gleichzeitig Aushandlungsprozesse der Erzählenden. Sie geben nicht nur subjektive Wahrnehmungen der Erzählenden beispielweise von Räumen wieder, sie haben auch eine Funktion des Verstehens und Ordnens unserer Welt (Ricoeur 1984; 1986). Dadurch, dass Erzählen eine eingeübte Alltagskompetenz ist, bietet es den Stadtnutzerinnen/-nutzern einen anderen Zugang zu Forschung als sonst im hierarchischen Machtgefälle zwischen Wissenschaft und Beforschten. Gleichzeitig müssen dafür von den Wissenschaftlerinnen/Wissenschaftlern Rahmenbedingungen geschaffen werden, beispielsweise um Vertrauen zu schaffen oder fehlende Sprachkenntnisse zu überbrücken.

Neben den Funktionen des Erzählens ist die Auseinandersetzung mit Erzählungen als Produkt ein zentrales Element der Erzählforschung. Für das Verständnis von Erzählungen gibt es jedoch keine eindeutige Definition, sie kann immer nur für den spezifischen Kontext aufgestellt werden. Wir konzentrieren uns auf Erzählungen von Stadtnutzerinnen/-nutzern über sich und ihren Stadtteil. Hier erweist sich der Rückgriff auf das Konzept der Small Stories (Bamberg/ Georgakopoulou 2008) als zielführend. Small Stories bilden das Pendant zu ,großen Erzählungen' und sind ein Oberbegriff für unterrepräsentierte Erzählungen, wie z. B. von laufenden Ereignissen, zukünftigen, hypothetischen oder gemeinsam erlebten Ereignissen (Bamberg/Georgakopoulou 2008: 381).

In den vergangenen drei Jahrzehnten wurde in der Planungswissenschaft die Beziehung zwischen Erzählungen und Planung untersucht (Mandelbaum 1991; Throgmorton 1992; Eckstein/Throgmorton 2003; Sandercock 2003; Throgmorton 2003; Finnegan 2004; Altrock/Huning/Peters 2006; Sandercock/Attili 2010; van Hulst 2012). Nach Sandercock (2003: 18f.) produzieren Geschichten wichtiges Wissen über Städte: Im Sinne einer kommunikativen oder 
partizipativen Planung kann Erzählen eine Methode sein, die Menschen einlädt, Geschichten über ihre Lebenswelt aus unterschiedlichen Perspektiven zu erzählen und dadurch dominanten Erzählungen etwas entgegenzuhalten und alternative Lesarten zu eröffnen. Wie die Gesprächspartner/-innen sich im Kontext ihres Stadtteils positionieren und welche Identität sie sich selbst zuschreiben, gehört zum impliziten Wissen und lässt sich nicht direkt abfragen, aber durch Erzählungen produzieren: „When we tell stories, write stories, or compose stories [...] we are using narrative structures in ways that not only communicate but also shape how we position ourselves and others" (McVee/Boyd 2015: 18). Die Art und Weise, wie wir von der Stadt erzählen, wirkt sich zudem konstitutiv auf unsere Wahrnehmung von Städten aus und beeinflusst die Entscheidungen, die wir treffen, sowie die Art und Weise, wie wir handeln (Sandercock 2003: 12).

Erzählen als methodischer Zugang kann als niedrigschwelliger Ansatz verstanden werden, durch den Menschen ihre Geschichten und individuellen Perspektiven in eigenen Worten erzählen können (Vacchelli 2018: 69f.). Gleichzeitig ist die Abgrenzung von (klassischer) Erhebung im Forschungskontext, Ko-Produktion von transformativem Wissen und traditioneller Beteiligung in der Planungspraxis nicht trennscharf zu definieren, da Schnittmengen existieren. Die Erzählsituationen knüpfen an narrative und partizipative Forschungsmethoden an, modifizieren sich jedoch für den INTERPART-Kontext: Die Unterschiede liegen weniger in Oberflächenmerkmalen der Methode als in der grundlegenden Ausrichtung bei ihrer Anwendung. Hier geht es nicht allein um reine Datenerhebung und -auswertung zur Beantwortung von Forschungsfragen ${ }^{3}$, sondern auch um das Schaffen eines Lernorts zur Erarbeitung einer Wissensbasis, auf der die Weiterentwicklung des ReallaborKonzeptes gründet.

\section{Zwischenfazit}

Dass Reallabore Forschung und Partizipation simultan gerecht werden müssen, sehen wir als Anlass, Methoden der klassischen empirischen Sozialforschung weiterzuentwickeln. Auf der Grundlage der Erkenntnisse aus den theoretischen Auseinandersetzungen scheinen bewusst gestaltete Erzählsituationen ein geeigneter methodischer Ansatz zur konzeptionellen Ausarbeitung des Reallabors zu sein, indem sie der transdisziplinären Ko-Forschung Zugang zum
Forschungsthema auf einer Ebene eröffnet, die am eigenen Alltagswissen anknüpft und eine gemeinsame Sprachebene schafft.

Erzählsituationen können außerdem dazu beitragen, zwischenmenschliche Machtgefälle zwischen Wissenschaft und Ko-Forschung auszugleichen, sofern sich die Wissenschaftler/-innen demgegenüber offen zeigen und ihren eigenen Bias bewusst reflektieren. Zentral ist dabei die inhaltliche und gestalterische Konzeption der Erzählräume. Gleichzeitig sind dies die Herausforderungen in der konkreten Umsetzung und darauffolgenden Auswertung.

Um dem zu begegnen, bildet die Konzeption der Erzählsituationen aus den Perspektiven der Design- und Raumforschung einen zentralen Fokus. Wir diskutieren dazu die Gestaltung physischer Erzählräume, die zur Herstellung einladender, offener und zugleich geschützter Erzählsituationen beitragen sollen - ausgehend von der Hypothese, dass die räumliche Gestaltung wesentlich dazu beiträgt, dass Teilnehmer/-innen mitmachen, Vertrauen gewinnen und das Alltagswissen einbringen. Weiterhin reflektieren wir die Grenzen narrativer Vorgehensweisen im Kontext unseres Reallabors.

INTERPART will mit den Erzählangeboten Voraussetzungen für Partizipation schaffen, eine gemeinsame Basis herstellen, die Relevanz der Fragestellung eruieren bzw. sie gemeinsam weiterentwickeln. Der transformative Charakter ergibt sich (auch) dadurch, dass die Teilnehmer/-innen im Erzählen erfahren können, dass sie etwas zum Thema Stadtentwicklung beitragen können, dass ihre Stimme wichtig ist, sie Partizipation an Stadtentwicklung genauer bzw. auf ihre Person bezogen verstehen und dadurch die Wahrscheinlichkeit künftiger Beteiligung erhöht werden kann. Durch die begrenzte Laufzeit des Reallabors können wir allerdings nicht überprüfen, inwiefern dieser Effekt tatsächlich eintritt.

Die im Folgenden dargestellten Erzählsituationen dienen der Vertrauensbildung und dem Einstieg in den Dialog mit Menschen vor Ort. Im Idealfall erfahren die an den Gesprächen Beteiligten Selbstwirksamkeit als nachhaltigen Effekt zum Abbau von Barrieren gegenüber Teilhabe. Gleichzeitig bezieht sich das Erkenntnisinteresse inhaltlich auf die Ausgestaltung interkultureller Dialogräume. Die Erzählsituationen stehen am Anfang des Reallabor-Prozesses und neben ihnen folgen weitere Ko-Produktionen - mit Akteuren aus der Zivilgesellschaft und darüber hinausgehend. Die Erzählungen und die dadurch geschaffene Wissensbasis fließen also nur als ein Part in die Beantwortung der Forschungsfragen ein.

\footnotetext{
3 Die Beantwortung von Forschungsfragen wurde im komplexen Methoden-Mix des Projekts allerdings ergänzend ebenfalls vorgenommen.
} 


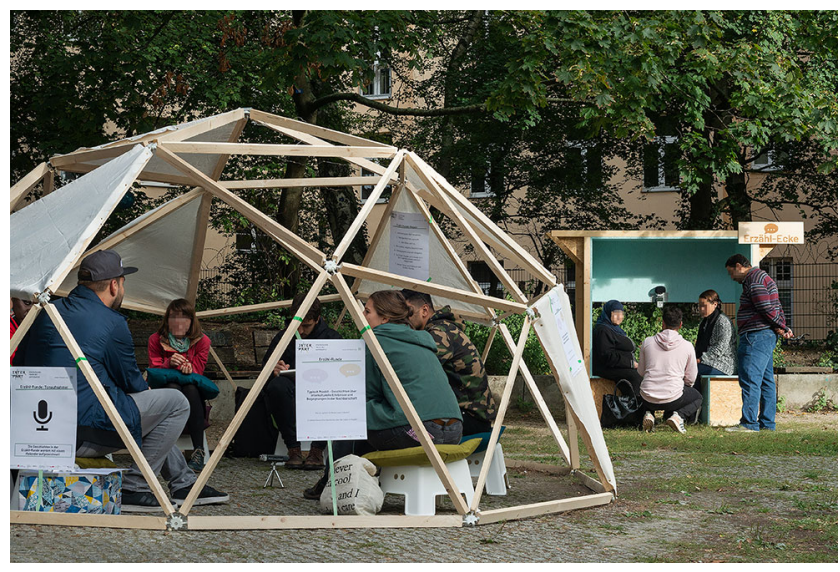

Abbildung 1 Die Erzählrunde (Vordergrund) und die Erzählecke (Hintergrund) während der Vor-Ort-Intervention in Berlin-Moabit, auf dem Platz an der Ecke Salzwedeler Straße/Quitzowstraße (c) UdK Berlin, Fotograf: Michael Shenbrot

\section{Erzählen im Forschungsprojekt INTERPART}

INTERPART verfolgt einen breiten methodischen Ansatz, aus dem in diesem Beitrag innerhalb des Reallabor-Formats die sogenannten Vor-Ort-Interventionen herausgegriffen werden, von denen jeweils zwei in den Fallbeispielen (Stadtteile Berlin-Moabit und Wiesbaden-Biebrich) stattfinden. Der Fokus der Vor-Ort-Interventionen liegt in der Kombination von analogen und digitalen Partizipationsformaten, die im öffentlichen Raum Menschen in ihren alltäglichen Lebensräumen ,abholen'. Anders als bei klassischen Partizipationsverfahren steht kein konkretes Planungsvorhaben im Vordergrund. Die Vor-Ort-Interventionen sind als Beginn eines Dialogs mit lokalen Akteuren und der Prozess als ergebnisoffen und als gemeinsamer Lern- und Forschungsprozess angelegt. Die Erzählräume sind Bestandteil der Vor-Ort-Interventionen, die in beiden Städten auf öffentlichen Plätzen stattfinden, die zweite Vor-Ort-Intervention in Wiesbaden wird allerdings wetterbedingt in ein öffentliches Gebäude verlegt.

Neben spielerischen und kulinarischen Angeboten und einer hybriden Benutzerschnittstelle (eine multilinguale, interaktive Installation), die Menschen zu einem Dialog auffordert, dienen partizipative Formate des Erzählens in eigens dafür gebauten Erzählräumen Stadtnutzerinnen/-nutzern dazu, im Sinne eines Ortes gesellschaftlicher Aushandlungen Meinungen und Erzählungen über das Zusammenleben in ihrer Nachbarschaft als geteiltes Wissen zu produzieren (vgl. Abbildung 2). Die Erzählräume bezeichnen wir als Erzählecke und Erzählrunde und bauen sie jeweils im öffentlichen Raum auf. Die Erzählecke dient dazu, mit den Stadtnutzerinnen/-nutzern in einen Austausch über ihren Stadtteil zu kommen, um gemeinsam Wissen über den

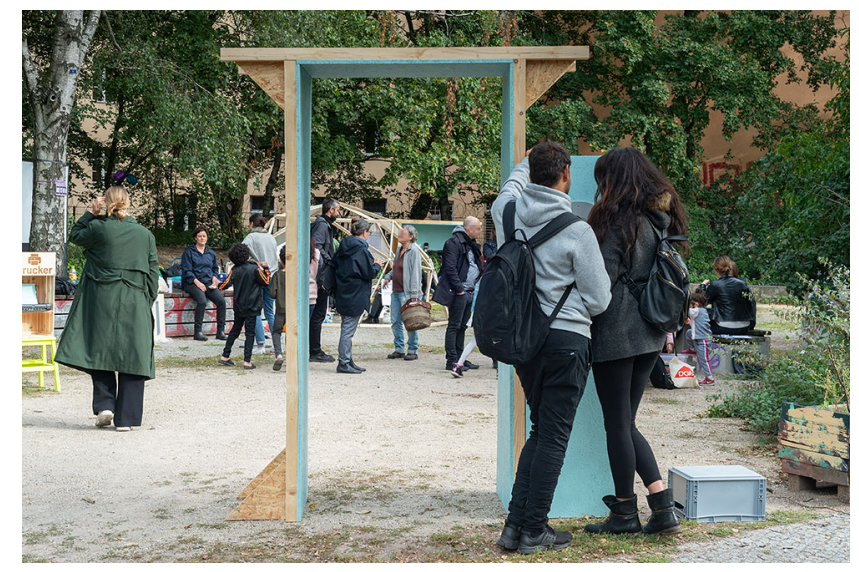

Abbildung 2 Eingang zum temporären Interventionsraum der Vor-Ort-Intervention in Berlin-Moabit

( ) UdK Berlin, Fotograf: Michael Shenbrot

Stadtteil, das Zusammenleben und Erfahrungen mit Partizipation zu produzieren. Im Rahmen der zweiten Intervention bieten wir zusätzlich eine Erzählrunde an, ebenfalls ein physischer Raum. Dabei sind Stadtnutzer/-innen eingeladen, sich in einer Gesprächsrunde über das Zusammenleben im Stadtteil auszutauschen und mit uns Wissenschaftlerinnen ein geteiltes ,Bild“ dieses Zusammenlebens zu verhandeln.

\subsection{Erzählräume gestalten}

In diesem Abschnitt erörtern wir sowohl die Gestaltung der physischen Erzählräume und die Gestaltungsprinzipien, die den Entscheidungen zugrunde liegen, als auch die sprachlich-konzeptionelle Ebene der Erzählsituationen. Da sie sich gegenseitig bedingen, erfolgt zunächst die Darstellung beider Dimensionen ineinander übergreifend. Die konkrete Ausgestaltung von Erzählecke und Erzählrunde soll schließlich Einblicke in experimentelle Formate und ihren Mehrwert im Kontext Reallabor gewähren.

Die sprachliche Ebene der Erzählsituationen umfasst die Konzeption aus planungswissenschaftlicher Sicht und auf der Basis qualitativer, insbesondere narrativer Forschungsmethoden (vgl. Schütze 1983; Flick 2011). Die Gespräche in der Erzählecke dienen dazu, mit den Menschen vor Ort in Kontakt zu kommen. Thematisch geht es um Inhalte aus den Forschungsfragen des Projektes: Im Zentrum steht die gemeinsame Rekonstruktion, wie Stadtnutzer/-innen das interkulturelle Zusammenleben im Quartier aushandeln, welche Zuschreibungen dabei von Bedeutung sind und welche sozialen Beziehungsmuster die Akteure knüpfen. Uns kommt es darauf an, „migrationsgeprägte Quartiere weniger von außen mit Daten und Statistiken im Vergleich mit anderen Quartieren oder den gesamtstädtischen Durchschnittswerten wahrzunehmen, als sich auf die Suche zu machen 
nach Alltagssituation und Lebenslagen der dort lebenden Menschen“ (Beer 2013: 47). Die Menschen in den VorOrt-Interventionen werden als Expertinnen/Experten ihres Viertels angesprochen. Die Ansprache unterschiedlicher Alters- und Geschlechtsgruppen können wir anhand sichtbarer Merkmale weitgehend sicherstellen. Der Einbezug von Menschen mit Migrationsgeschichte bzw. Flüchtlingsstatus ist nicht steuerbar, da dies anhand optisch wahrnehmbarer Merkmale nicht möglich ist und auf potenziell unzutreffenden Fremdzuschreibungen basieren würde. Diese Merkmale versuchen wir durch die entsprechenden Selbstbeschreibungen und ex post durch ergänzende Angaben zur Person zu rekonstruieren.

Die physisch-räumliche Gestaltung der Erzählsituationen entsteht vor dem Hintergrund der Frage, welche Möglichkeiten ein bewusst gestaltetes räumliches Setting für das Erzählen im Reallabor eröffnet. Der Gestaltungsprozess ist Ergebnis des Dialogs innerhalb des transdisziplinären Projektteams sowie intermediären Ko-Forscherinnen/-Forschern aus der Quartiersarbeit. Von der jeweiligen Spezifik des urbanen Kontextes geleitet, erfolgen die Entwicklung und das Bauen der physischen Erzählräume.

Die spezifischen Eigenschaften und Qualitäten der öffentlichen Räume in Berlin-Moabit und Wiesbaden-Biebrich bilden den Ausgangspunkt für das Design der Erzählecke und der Erzählrunde. Im Fall von Moabit basiert die Entscheidung für die Positionierung der Erzählräume im östlichen Teil des Platzes unter anderem auf der Beobachtung, dass Passantinnen/Passanten nachmittags und abends den Platz aus westlicher Richtung von der nahegelegenen S-Bahn-Station in Richtung Wohngebiet überqueren. Innerhalb des Ensembles der partizipativen Angebote auf dem Platz platzieren wir das ,Eingangstor ‘ und die Erzählecke so, dass sie sich den Menschen, von der S-Bahn kommend, hinwenden. So wirken sie einladend und offen, ohne die Laufwege zu blockieren oder ihnen zu nah zu sein (vgl. Abbildung 1).

Bei der Gestaltung und Platzierung achten wir außerdem darauf, welche Raumqualitäten für eine Erzählsituation wichtig sind: Wie werden Geräusche in den einzelnen Platzbereichen wahrgenommen? Inwiefern würden sie eine Gesprächssituation positiv oder negativ beeinflussen? Wo fühlt man sich eher geschützt, wo exponiert? Das östliche Ende des Platzes liegt am weitesten von der stark befahrenen, geräuschintensiven Putlitzbrücke entfernt und eignet sich daher auch deswegen gut für die Platzierung der Erzählräume. Letztlich wird die Position der Erzählräume auf dem Platz auch durch die restlichen Elemente der Vor-OrtIntervention strukturiert (vgl. Abbildung 2).

Die Mitarbeiter/-innen unseres lokal verankerten Projektpartners in Moabit gehen selbst aktiv auf Besucher/-innen der Vor-Ort-Intervention zu, um sie in die Erzählräume ein- zuladen. Sie agieren als „Gatekeeper“ (Müller 2018: 61f.) und tragen zu einer vertrauensvollen Atmosphäre auf dem Platz bei. Die Anwesenheit von Sprachmittler/-innen, mehrsprachiges Informationsmaterial sowie eine mehrsprachige interaktive Installation sollen darüber hinaus eine Kultur der Mehrsprachigkeit und eine in Bezug auf Vielheit offene Haltung signalisieren.

Die Formate Erzählecke und Erzählrunde bedienen die Ansprüche an unterschiedliche Situationen: Während durch die Konstruktion der Erzählecke eine halbintime Atmosphäre entsteht, bei der ein/e Wissenschaftler/-in und ein/e Bewohner/-in sich in einer Zweierkonstellation gegenübersitzen, lädt die Erzählrunde mehrere Teilnehmer/-innen ein, sich am Erfahrungsaustausch (gegebenenfalls auch nur punktuell) zu beteiligen. Die Art, wie sich Wissenschaftler/-innen und Stadtnutzer/-innen in der Erzählecke und der Erzählrunde gegenübersitzen (einander zugewandt, auf der gleichen Ebene, mit oder ohne Tisch, in einer Runde usw.), soll dabei zu einer vertraulichen Atmosphäre führen und zu einer Ko-Produktion möglichst auf Augenhöhe beitragen.

Unterstützt wird der Versuch eines Gesprächs auf Augenhöhe durch die Ansprache der Stadtnutzer/-innen als Expertinnen/Experten ihrer Lebenswelt. In dieser Situation ist uns bewusst, dass wir Wissenschaftlerinnen den Stadtnutzerinnen/-nutzern gegenüber in einer mächtigeren Position verbleiben, da wir das Thema des Projekts kennen und auf einen Gesprächsleitfaden zurückgreifen können. Das Machtgefälle kann sich verstärken, wenn die Stadtnutzer/-innen sich durch Selbst- und/oder Fremdzuschreibung als Menschen mit Migrationsgeschichte in der sozialen Welt positionieren und Differenzen zur Wissenschaftlerin als Vertreterin der weißen, privilegierten Mittelschicht wahrnehmen. Andere Stadtnutzer/-innen können dies genau gegenteilig, nämlich im Sinne von Gleichheit empfinden: So könnten sich Frauen ohne Migrationsgeschichte eher mit der Wissenschaftlerin als ebenfalls weiße Frau identifizieren. Zwischen Stadtnutzer/-innen und Wissenschaftlerin gibt es also die Wahrnehmung von Gleichheit oder Differenz aufgrund von Geschlecht, Herkunft und Milieu. Die Annahmen über Gleichheit und Differenz beeinflussen, welche Informationen die Wissenschaftlerin von Frauen erhält, aber auch, wie sie die Daten interpretiert. Dies wird im Auswertungsprozess reflektiert.

In der Erzählecke orientieren wir uns am episodischen Interview (Flick 2011). Da wir ein hierarchisches Machtgefälle zwischen den Menschen aus Wissenschaft und Stadtteil vermindern möchten, bereiten wir mögliche Gesprächsthemen vor und sind gleichzeitig offen für die Erzählungen der Stadtnutzer/-innen. Im Mittelpunkt steht die individuelle Positionierung zu eigenen Erfahrungen oder Erfahrungen mit Migration, Genderrollen oder Lebenssti- 
len der Stadtnutzer/-innen in ihrer Nachbarschaft sowie ihre Erlebnisse mit analogen und digitalen Partizipationsmethoden. In Berlin-Moabit liegen den Wissenschaftlerinnen/ Wissenschaftlern die Themen Diversität und Differenz, Partizipationsbarrieren und Crossmedialität als Leitfaden vor, der Einstieg erfolgt über einen Erzählimpuls. In WiesbadenBiebrich verstärken wir den Effekt der von den Stadtnutzerinnen/-nutzern selbstgewählten Perspektive und bieten ein sogenanntes Erzählmenü an. Dies beinhaltet sechs Themenvorschläge, aus denen die Stadtnutzer/-innen auswählen können. Die Erzählungen beinhalten Meinungen und Geschichten über das Zusammenleben in einem Quartier als Ort gesellschaftlicher Aushandlungen. Ausgerichtet auf ein persönliches Gespräch kann die Erzählecke zu einer ersten Vertrauensbildung zwischen den Wissenschaftlerinnen und Stadtnutzerinnen/-nutzern beitragen. Unterstützt wird die Vertrauensbildung an beiden Orten durch die Hilfe von Sprachmittlerinnen/-mittlern.

Die Sitzposition bei der räumlichen Konstruktion der Erzählecke ist so gewählt, dass sich die Gesprächspartner/-innen auf identischen Sitzflächen gegenübersitzen. Den Sitzabstand haben wir im Konstruktionsprozess iterativ überprüft und angepasst - in dem Bewusstsein, dass sich die Sprechenden während der Intervention fremd sein würden. Das Mikrofon zur Aufzeichnung der Erzählungen ist bewusst an der Seitenwand und nicht zwischen den Gesprächspartnerinnen bzw. -partnern platziert, damit die Technik keine Barriere zwischen den Körpern darstellt. Formal ist die Erzählecke als eine Art Kabine gestaltet, die nach oben, zum Rücken und zu einer Seite hin geschlossen ist. Die Wände können zum Anlehnen genutzt werden und bieten auch Schutz vor Umgebungslärm. Die Öffnung zur einen Seite ist wichtig, damit Menschen im Interventionsraum sehen können, dass dort ein Gespräch stattfindet. Sprachmittler/-innen können einen Hocker heranziehen und sich zum Gespräch dazusetzen. Anhand der Konstruktionsart, des Materials und der Farbe des Innenraums der Erzählecke stellen wir eine visuelle Verbindung zu den weiteren Elementen der Intervention her (Tor mit Klingelinstallation, an der in der gewählten Sprache mit dem ,Platz kommuniziert werden kann; Drucker, der die Eingaben an der Klingel in fünf Sprachen ausdruckt; Erzählrunde; Erzählecke; vgl. Abbildung 2).

Bei der zweiten Vor-Ort-Intervention wird die Erzählrunde als zusätzliches narratives Format in Weiterentwicklung der Zweier-Gesprächssituation der ersten Vor-Ort-Intervention erprobt. Die Erzählrunde folgt den Prinzipien des Erzählsalons, bei dem die Menschen aus dem Stadtteil über ein vorgegebenes, aber relativ breit angelegtes Thema ins Gespräch kommen (Richter/Rohnstock 2016). Zu beobachten gilt, inwiefern es gelingt, einen vertrauensvollen Gesprächsraum zu schaffen, in dem sich die Diversität der
Bevölkerung im Quartier widerspiegelt und wie dabei die eigene Zugehörigkeit zu Räumen und Nachbarschaften definiert wird: ,the act of constructing an identity as a member of a residential neighborhood" (Ball-Rokeach/Kim/Matei 2001: 394). Im Unterschied zur Gruppendiskussion handelt sich um einen Erzählraum, den die Teilnehmenden im Laufe des Gesprächs verlassen können bzw. neu hinzukommen können. Die Moderation übernehmen im Sinne der Ko-Forschung eine Wissenschaftlerin und eine Multiplikatorin aus dem Stadtteil gemeinsam. Letztere kann den Teilnehmenden gegenüber eine Vertrauensperson darstellen und helfen, das Machtgefälle zwischen Wissenschaftlerinnen und Stadtnutzerinnen/-nutzern auszugleichen. Die Erzählungen entwickeln sich - im Unterschied zur Erzählecke - weniger zwischen Stadtnutzerinnen/-nutzern und Wissenschaftlerin, sondern stärker zwischen den Stadtnutzerinnen bzw. Stadtnutzern.

Eine geodätische Kuppel - ein Gebilde aus dreieckigen, hölzernen Steckverbindungen, das an eine halbierte Weltkugel erinnert (vgl. Abbildung 1) - bildet die räumliche Gestalt für die Erzählrunde. Einige der dreieckigen LeerRäume der Kuppelkonstruktion bedecken wir mit Stoff, um einen durchlässigen Erzählraum zu erhalten, der Schutz bietet und von außen trotzdem einsehbar ist. Wir gehen davon aus, dass es Menschen leichter fällt, sich in die Erzählrunde zu setzen, wenn sie den Raum als offen und dennoch abgegrenzt empfinden und selbstständig entscheiden können, wann sie kommen und gehen. Die Ko-Forscher/-innen sitzen sich auf Hockern im Kreis gegenüber, damit beziehen sich ihre Sitzpositionen aufeinander. Die Sitzhöhe der Hocker $(\mathrm{ca} .30 \mathrm{~cm})$ ist so gewählt, dass sich die Sitzenden auf einer anderen Ebene als die Menschen außerhalb der Kuppel befinden.

\subsection{Erzählungen aus dem Reallabor INTERPART}

Im Folgenden wollen wir auf ausgewählte Erkenntnisse aus der Anwendung der experimentellen Erzählformate zur Partizipation am Reallabor eingehen. In der ersten Vor-OrtIntervention stellen wir unter anderem die Erzählecke auf und laden die Stadtnutzer/-innen ein, über ihren Stadtteil und Erfahrungen mit Beteiligung zu erzählen.

In der Erzählecke in Biebrich werden in verschiedenen Erzählungen über das Zusammenleben im Stadtteil kontrastierende Storylines deutlich. ${ }^{4}$ Hier gibt es Äußerungen, die durchaus rassistische Züge tragen bzw. mit Othering-

\footnotetext{
${ }^{4}$ Grundlage bilden die Transkripte der Gespräche mit den Stadtnutzerinnen/-nutzern aus Wiesbaden-Biebrich und Berlin-Moabit.
} 
Mechanismen ${ }^{5}$ arbeiten. Eine Frau berichtet von einer Diskussion im Stadtteil über den Wandel, den einige Menschen wahrnehmen: „Wie früher, schade, dass es nicht mehr ist wie früher. Der Metzger ist weg, da ist jetzt ein türkischer Supermarkt, ne? So, wo sind die traditionellen deutschen Geschäfte?" Eine weitere Gesprächspartnerin greift dieses Thema in der Erzählecke auf. Sie erzählt: „Manche sagen so plakativ, die eine Straße gehört den Griechen und die andere den Türken“. Sie distanziert sich von den Wahrnehmungen, geht aber nicht auf ihre eigene Position ein. Ein solches Denkmuster lässt sich als Othering beschreiben.

Es gibt aber auch Gegenerzählungen, in denen der Wandel positiv beschrieben wird. „Für uns hat sich alles zum Positiven entwickelt. Wir finden hier alles Wichtige“, ist ebenfalls eine Aussage zur Entwicklung der EinkaufsstraBen. In diesem Fall verläuft die Trennlinie der Perspektiven entlang dem Vorhandensein bzw. Nichtvorhandensein einer Migrationsgeschichte. Diese Konfliktlinien wollen wir nicht nur als rassistische Denkmuster stehenlassen, sondern die tieferliegenden Ängste vor den Veränderungen und den Verschiebungen der Gestaltungsmacht im Stadtteil mitberücksichtigen. Die Abweichungen der Darstellungen zeigen aber vor allem, wie wichtig es ist, Gegenerzählungen nicht nur zuzulassen, sondern gerade in Partizipationsprozessen auch zu suchen, um der dominanten Sichtweise im Stadtteildiskurs einer vermeintlichen Mehrheit Counterstories entgegenzusetzen und ihnen Gehör zu verschaffen (Solorzano/ Yosso 2001).

Diese Art von Erzählungen zeigt, wie sich Menschen selbst einordnen, welcher Gemeinschaft sie sich zugehörig fühlen und von wem sie sich abgrenzen. Zudem wird deutlich, wie Räume, in denen sich die Stadtnutzer/-innen bewegen, zu Identifikationsräumen oder eben zu fremden Räumen werden. Die Herausarbeitung der Erzählung über den Verlust von Bezugspunkten von Menschen ohne Migrationsgeschichte durch migrantische Ökonomien macht in Verbindung mit einer abwertenden Haltung gegenüber Migration deutlich, dass Othering-Prozesse und Rassismus bei Themen der Stadtentwicklung eine Rolle spielen und in Partizipationsprozessen reflektiert und aktiv begegnet werden müssen.

In den Erzählungen zeigt sich, inwieweit Aushandlungsprozesse im Stadtteil über die Ausgestaltung interkultureller Dialogräume schon seit einigen Jahren stattfinden. Stadtnutzer/-innen mit Migrationsgeschichte betonen, dass

\footnotetext{
5 Othering bedeutet übersetzt „Verandern“ und beinhaltet gezieltes Ansprechen von Migrantinnen und Migranten als Gruppe mit ,anderen“ Interessen und Bedürfnissen gegenüber einer Mehrheitsgesellschaft, was dazu führt, dass auch in der Partizipation Machtverhältnisse auf einer strukturellen Ebene reproduziert werden (Römhild 2017: 65).
}

für ein interkulturelles Miteinander beide ,Seiten ‘ gefordert sind, um ins Gespräch zu kommen. Durch ihre Erzählungen machen sie deutlich, welche Anstrengungen sie unternehmen müssen, um akzeptiert zu werden. Eine Erzählung greifen wir als Beispiel heraus.

Also ich lebe seit ungefähr 28 Jahren hier in Biebrich. Ich war, als wir hierhergezogen sind, fünf Jahre alt. Wir kamen von Stadtteil Y nach Stadtteil Biebrich. Aber als wir hier einziehen wollten, waren alle gegen uns. Weil wir Ausländer waren. [...] Das waren drei Familien, zwei aus Syrien, eine aus der Türkei. Aber wir waren drei christliche Familien. Und als wir einziehen wollten, haben kaum Ausländer hier in Biebrich gelebt. Also wir waren eine der ersten Ausländer, die nach Biebrich gezogen sind. Und alle waren dagegen. Also es wurden Unterschriften gesammelt, dass hier keine Ausländer reindürfen. Und wir haben dann mit den, es gab zwei Gemeinden, die Katholische Gemeinde und die Evangelische Gemeinde. Wir haben mit den Pfarrern, also die Eltern von uns, sage ich mal, es gab auch Dolmetscher damals, weil wir die Sprache nicht beherrschten, die haben halt übersetzt, dass wir aus christlicher Gemeinde kommen. Und dann hieß es: „Ja, es gibt zwei Gemeinden, katholische und die evangelische. Ihr sollt euch mit denen in Verbindung setzen. "Und dann hieß es, ja, wir können uns einmal treffen. Dass man sich dann vorstellt. Und vielleicht kann man doch da was machen. Das Haus wurde fotografiert, die Familien wurden fotografiert. Wir kamen in die Zeitung rein - „Das ist unmöglich. Also es dürfen keine Ausländer rein. “ Und als wir uns dann getroffen haben, es wurde eine Veranstaltung veranstaltet. Jeder sollte eine Kleinigkeit mitnehmen, dass man bisschen in Kontakt kommt. Und wir wurden dann auf einmal so herzlich aufgenommen, weil die dann gemerkt haben, ja, das sind doch keine schlimmen Menschen oder das sind ganz, normale Menschen. Wir sind auch eine Minderheit, wir sind christliche Aramäer. Und als die uns dann kennengelernt haben, hat sich die ganze Seite umgewandelt. Wir wurden dann herzlich willkommen hier in Biebrich.

Die Erzählerin als erzählendes Ich (Lucius-Hoene/Deppermann 2004: 172, 178) positioniert sich als Person, die schon lange im Stadtteil lebt und sich sehr wohlfühlt. Die handelnde Person der Erzählung als erzähltes Ich (Lucius-Hoene/ Deppermann 2004: 172, 178) ist jedoch nicht die Erzählerin selbst, sondern bezieht sich auf die Kinder der drei Familien, die vor 28 Jahren in den Stadtteil ziehen. Die Fremdbezeichnung „Ausländer“ übernimmt sie für das erzählte Ich und macht mit dem Verb „waren“ im Präteritum 
deutlich, dass sie sich damit nicht mehr identifiziert. Durch die Entwicklung, die sie durch ihre Erzählung beschreibt, wird das erzählte Ich, also die drei Familien, von „Ausländern“ zu „,normalen Menschen“. Die Familien sind die handelnden Charaktere - in der Erzählung, aber auch in dem Prozess der Integration, von dem die Frau erzählt. Sie übernehmen den aktiven Part und verändern so die Nachbarschaft, die sich zunächst stark gegen die Integration wehrt. Der wichtigste Anknüpfungspunkt der Familien sind die beiden Kirchengemeinden. Dies kann als eine Abgrenzung zu den muslimischen Geflüchteten gelesen werden, die seit 2015 verstärkt nach Deutschland immigrieren.

Durch ihre Erzählung erfahren wir als Wissenschaftlerinnen mehr darüber, welche Geschichten sich hinter den statistischen Zahlen verbergen, die den Stadtteil als besonders multikulturell im Vergleich zur Gesamtstadt beschreiben (vgl. z. B. SEG 2020). Es wird neues Wissen produziert über die Vorgänge der Integration aus der Sicht von Menschen, die selten diesbezüglich zu hören sind. Im Zusammenspiel mit der Tatsache, dass die Wissenschaftlerin als Zuhörerin weiß ist, (ko)produzieren Stadtnutzerin und Wissenschaftlerin in dem Moment gemeinsam eine Counterstory (Solorzano/Yosso 2001: 475) zu populistischen Geschichten im Sinne des assimilierenden Integrationsverständnisses. Das heißt, sie müsste einer Person mit gleichem Erfahrungshorizont diese Erzählung nicht mitgeben, um ihre Perspektive nachvollziehbar zu machen. Counter-Storytelling ist eine Methode, die Geschichten jener Erfahrungen von Menschen zu erzählen, die nicht oft erzählt werden, und ein Werkzeug für Analyse und Infragestellung der Geschichten derjenigen, die in den Machtpositionen sind und deren Geschichte einen ,natürlichen' Teil des vorherrschenden Diskurses ausmachen (Solorzano/Yosso 2001: 475).

Als Weiterentwicklung der Erzählecke und zusätzliches Element der zweiten Vor-Ort-Intervention orientiert sich die Erzählrunde an der Methode des Erzählsalons. Methodisch werden im Erzählsalon zwei Erhebungsverfahren aus der Sozialforschung miteinander verknüpft: das narrative Interview und die Gruppendiskussion (Richter/Rohnstock 2016: 85). Darüber hinaus dienen Erzählsalons als strategisches Mittel der Vertrauensbildung und der Selbstermächtigung von sozialen Gruppen und der Gemeinschaft (Richter/ Rohnstock 2016: 86). Anknüpfend an die Erzählecke geht es darum herauszufinden, über welche Themen der Stadtentwicklung und des Zusammenlebens gesprochen wird, wenn sich das Gespräch aus einer diversen Nachbarschaftsgruppe zusammensetzt, sowie wie Menschen miteinander kommunizieren wollen, welche Emotionen in einer gemeinsamen Gesprächssituation zu Tage treten und wie ein interkultureller Dialog niedrigschwellig und inklusiv gestaltet werden könnte. Das Thema der Erzählrunde soll die Menschen dazu anregen, ihre eigenen interkulturellen Erfahrungen zu schildern, durch ihre Geschichten über die Gestaltung eines interkulturellen Dialograums ins Gespräch zu kommen und geteiltes Wissen zu generieren. In diesem lautet dann auch das Gesprächsthema in Berlin: ,Typisch Moabit - Geschichten über interkulturelle Erlebnisse und Begegnungen in der Nachbarschaft“.

Als spezifische Form der Kommunikation können Erzählungen der Repräsentation individueller und kollektiver Identitätsbildungsprozesse der Gruppenbildung dienen und den Zusammenhalt innerhalb einer Gemeinschaft fördern (Sommer 2017: 257). Eine Erzählgemeinschaft kann, wie die Erzählrunde in der Vor-Ort-Intervention, temporär zusammenfinden und sich auch auf Nachbarschaften konzentrieren (Sommer 2017: 257). Die Teilnehmer/-innen einer Erzählgemeinschaft unterscheiden sich hinsichtlich ihrer Rollen während der Erzählrunde: Manche tun sich aktiv als Erzähler/-innen hervor und gestalten den narrativen Diskurs. Diese Rolle übernimmt in der Erzählrunde beispielsweise eine Frau ohne Migrationsgeschichte, die sich zu Beginn mit dem Thema Gentrifizierung als aktive, informierte Stadtnutzerin positioniert: Sie beobachtet die Gentrifizierungsprozesse im Stadtteil und steht ihnen kritisch gegenüber. Mit der Einführung dieses Themas entwickelt sich eine zentrale Storyline über den Einfluss der Gentrifizierung im Stadtteil, zu dem fast alle Teilnehmer/-innen etwas erzählen. Sie entwickeln damit ein kollektives ,Wir - diejenigen, die schon lange im Stadtteil wohnen und die Veränderungen wahrnehmen, und die anderen, die neu in den Stadtteil ziehen und Teil dieser Veränderungen sind. Auch hier lässt sich erkennen, wie Wissen koproduziert wird, auch zwischen den Stadtnutzerinnen/-nutzern selbst.

Die Dokumentation der Daten erfolgt per Audioaufnahme, aber nur mit Zustimmung der Erzählenden anhand einer Einverständniserklärung in mehreren Sprachen. Im Sinne des Forschungsstils der Grounded Theory (vgl. Strauss/ Corbin 1997) erfolgt die Auswertung der Daten aus Erzählecke und Erzählrunde nach dem Prinzip des theoretischen Codierens, einem kombinierten Verfahren aus offenem und selektivem Codieren (vgl. Flick 2005). In Erweiterung des Vorgehens der Grounded Theory nehmen wir eine Analyse einzelner Small Stories vor. Es geht um das inhaltliche Erkenntnisinteresse über das Verständnis interkulturellen Zusammenlebens und Partizipationserfahrungen der Stadtnutzer/-innen. Darüber hinaus besteht Erkenntnisinteresse bezogen auf das Erzählen als partizipative Methode. Die Erzählsituationen sind jedoch nur ein Bestandteil zur Beantwortung der Forschungsfragen. Die Erkenntnisse aus den partizipativen Erzählsituationen fließen zunächst in den iterativen Forschungsprozess mit ein und tragen am Ende zu den Erkenntnissen des Gesamtprojektes INTERPART bei, wobei der Schwerpunkt auf der experimentellen methodischen Herangehensweise liegt, bei der das offene Erzählen 
im Vordergrund steht. Um die Ergebnisse der Erzählungen aus den verschiedenen Veranstaltungen zu ko-evaluieren, findet in der Endphase des Reallabors ein Validierungsworkshop statt.

\section{Reflexion zur Durchführung der Erzählsituationen}

Wir haben eingangs deutlich gemacht, dass der Reallaboransatz nach neuen Methoden verlangt, um seinem Anspruch an Transdisziplinarität, Partizipation und Transformation gerecht werden zu können. Ausgehend von unseren theoretischen Überlegungen haben wir in den Vor-Ort-Interventionen Erzählräume geschaffen, in denen Stadtnutzer/-innen über das Zusammenleben im Stadtteil erzählen konnten, ohne dass sie mit einem detaillierten Leitfaden konfrontiert wurden oder sich in einer abfragenden Situation wiedergefunden hätten. Sie wurden als Expertinnen und Experten ihrer Lebenswelt angesprochen und konnten selbst wählen, worüber sie erzählen und wie lange. Die bewusst gestalteten Erzählräume als experimentelles, partizipatives Format stellen somit eine Weiterentwicklung gängiger Methoden der empirischen Sozialforschung dar.

Jedoch begegneten uns auch Herausforderungen und Grenzen der Erzählsituationen in allen Phasen: bei der Konzeption, der Durchführung sowie der Auswertung. Das Reallabor forderte bei der Konzeption der Interventionen eine Kombination aus Forschungs- und Partizipationsmethoden. Zum einen ging es um die Forschungsfragen, zum anderen, sie gemeinsam mit Akteuren aus der Praxis zu bearbeiten. Im Fall des INTERPART-Projektes lag der Schwerpunkt zudem auf der Frage nach der Einbindung von Menschen, die in Partizipationsprozessen der Stadtentwicklung unterrepräsentiert sind. Die Herausforderung, genau diese Menschen dann für eine Teilnahme an einer Intervention zu gewinnen, war daher besonders hoch. Diese Herausforderung manifestierte sich in den partizipativen Erzählsituationen: eine wissenschaftlich methodische Sauberkeit und Transparenz der Datenerhebung zu ermöglichen und dabei zur Teilnahme anzuregen. Nicht ausreichend gelungen ist es in dem Kontext, die Stadtbewohner/-innen bereits beim Ko-Design der Erzählräume einzubinden. Die Gestaltung wurde zwar, soweit es möglich war, mit ihnen reflektiert, aber in zukünftigen Konzeptentwicklungen kann dieser Umstand durch ein offenes Design noch stärker als Moment der Ko-Produktion berücksichtigt werden.

Bei der Durchführung wurde insbesondere die Erzählrunde zur Herausforderung. Das freie Erzählen in der Gruppe - eine der Stärken des Formats - war gleichzeitig eine Herausforderung für die Wissenschaftlerinnen, konkrete Forschungsfragen mit der Offenheit zu spontanen Erzäh- lungen, die in eine unerwartete Richtung gehen, zu verbinden. Gerade für die Auswertung spielte eine Rolle, dass die erzählenden Personen ihre Geschichten auf ihr Gegenüber abstimmen (Norrick 2010: 136f.) und so geteiltes Wissen produzierten. Auch in der Erzählecke fand die Ko-Produktion zwischen Wissenschaftlerin als Zuhörerin und Stadtnutzer/-in als Erzähler/-in und situativ auch mit Sprachmittler/ -innen als Übersetzer/-innen statt. Die Rolle der Gestaltung und Konzeption der Situationen im Zusammenhang mit der Rolle der Wissenschaftler/-in bei der Auswertung musste dementsprechend im gesamten Verlauf des Reallabors transparent bleiben und reflektiert werden.

In Zusammenhang mit Ko-Produktion sind im Weiteren die Perspektiven von Hydén (2013) und Vacchelli (2018) zu berücksichtigen, die sich mit Embodiment (Verkörperung) und dem Einfluss des Körpers auf Erzählungen auseinandersetzen. Sie argumentieren, dass der Körper - einschließlich Stimme, Schweigen, Blick, Berührung, Geruch und Bewegungen - niemals neutral ist und dass sowohl Worte als auch Erinnerungen tief mit körperlichen Erfahrungen verbunden sind (Hydén 2013: 128 ff.). Hydén (2013: 126f.) fordert Wissenschaftler/-innen im Zuge dessen auf, sorgfältiger über die Körper sowohl der Erzählenden als auch der Zuhörenden und die ständige Interaktion zwischen ihnen nachzudenken.

Die körperlichen Erfahrungen der Wissenschaftlerin in den Erzählräumen scheinen die Position von Hydén zu bekräftigen: Die gleichberechtigte Sitzposition, in der sich Erzähler/-in und Wissenschaftlerin in der Erzählecke gegenübersaßen, zusammen mit den Seiten- und Rückenwänden, die die Gesprächssituation umschlossen, wurde von ihr als vertraute Atmosphäre beschrieben, die sich bei beiden Sprechenden in einer entspannten Körperhaltung äußerte und die es ihr als Wissenschaftlerin erlaubte, bewusster aus der Haltung der Be-Forscherin in eine Rolle der Ko-Forscherin zu wechseln. Aus dieser Erfahrung schließen wir, dass die ungewohnte körperlich-räumliche Forschungssituation noch während des Gesprächs einen Reflexionsprozess über die eigene Positionierung in der Wissenschaftlerin ausgelöst hat. Dieses durch den Erzählraum hervorgerufene Bewusstsein für die eigene Rolle und Haltung kann einen positiven Effekt auf die gemeinsame Kommunikationsebene von Wissenschaftlerin und Stadtnutzer/-in haben, da scheinbar ein eigenes räumlich-körperliches Bezugssystem zwischen den Sprechenden entstanden ist.

Einige Beobachtungen verweisen auf einen ähnlichen Effekt eines temporären Bezugssystems in der Erzählrunde: Die geometrische Raumstruktur, die die Erzählenden umgab, schien als schützender Rahmen den Fokus vom äußeren Geschehen weg und hin auf die Gruppe zu lenken. Dieser Effekt ist insofern interessant, als dass der Erzählraum sehr durchlässig gestaltet wurde, das heißt, er schirmte 
weder Geräusche ab, noch schützte er vor Blicken von außen. Im Gegenteil: Blicke von außen wurden ganz bewusst zugelassen, um Menschen zum Mitmachen zu animieren. Die Grundform der geodätischen Kuppel strukturierte den Raum als eine ,Runde', in der sich Menschen im Kreis gegenübersaßen.

\section{Projekterfahrungen in einem größeren Kontext}

Abschließend wollen wir unsere Projekterfahrungen in den größeren Rahmen der Diskussion um den derzeit vielfach angewandten und ebenso vieldiskutierten Reallaboransatz setzen, insbesondere mit Fokus auf die Verknüpfung von Partizipations- und Forschungsmethoden.

Zentrale Erkenntnisgewinne umfassen das Zusammenspiel von methodischer, thematischer und gestalterischer Konzeption, die realweltliche Verortung und gleichberechtigte Gesprächssituation. Wir konnten darstellen, dass sich der Mehrwert des Erzählens für die Ko-Produktion von Wissen nicht nur durch die methodische Konzeption, sondern gerade auch die räumliche Gestaltung ergibt. Dies bestätigt die eingangs benannte produktive Kooperation zwischen Design- und Raumforschung. Zudem hat die interdisziplinäre Herangehensweise neue Möglichkeiten bei der Ko-Produktion von Wissen eröffnet, Reflexionsprozesse bei der eigenen Positionierung der Wissenschaftlerinnen ausgelöst und wertvolle Verknüpfungen zwischen den Erzählungen und den realweltlichen Räumen hergestellt. Ebenso zentral ist die Auseinandersetzung mit dem lokalen Kontext vor Ort, die Verortung der Erzählformate im öffentlichen Raum als Lebenswelt der Stadtnutzer/-innen. Die Umgebung der Erzählräume fließt in die Erzählungen ein und schafft eine Erzählsituation, die anders als eine Interviewsituation der erzählenden Person Freiraum gibt, eigene Schwerpunkte zu setzen. Dies muss jedoch auch bei der Einordnung der Erkenntnisse berücksichtigt werden: An anderen Orten hätten wir vermutlich andere Geschichten gehört. Des Weiteren stellen die Erzählräume eine gleichberechtigtere Situation dar, als es sich üblicherweise in Forschungsprozessen zwischen Wissenschaft und Beforschten beobachten lässt.

Herausforderungen, die bleiben und weiteren Forschungsbedarf deutlich machen, beziehen sich auf die Erzählsituationen als Forschungs- und Partizipationsformate und auf die Auswertungsprozesse. Zum einen stellt die Doppelrolle für Wissenschaftler/-innen in einem Forschungsformat, welches gleichzeitig die Stadtnutzer/-innen zur Partizipation einlädt, eine Herausforderung dar, wie bereits in der Einleitung zu diesem Beitrag spezifiziert. Darüber hinaus liegt für INTERPART der Fokus auf Menschen, die in herkömmlichen Partizipationsprozessen der Stadtentwicklung unterrepräsentiert sind, sodass eine Partizipation dieser Bevölkerungsgruppe besonders schwierig ist. Da transformative Wissenschaft gemeinwohlorientierte Veränderungen in der Gesellschaft verfolgt, ist die nachhaltige Wirkung des Empowerments durch Erzählen interessant. Ein Vorgehen zur Erhebung der Frage, inwiefern die Teilnahme an den Erzählformaten nachhaltig zu einer stärkeren Beteiligung an der Stadtentwicklung geführt hat, bleibt als Hinweis für zukünftigen Forschungsbedarf bestehen.

Weitere Herausforderungen, die im Reallaborkontext zustande kamen, ergeben sich im Rahmen der Auswertung von narrativem Datenmaterial, um beispielsweise den Einfluss von Positionierungen der Wissenschaftlerinnen, aber auch der Erzählorte auf die Ko-Produktion zu berücksichtigen. Dies verlangt eine intensive Reflexion über den gesamten Prozess. Im Sinne der Ko-Forschung werden im weiteren Verlauf des Reallabors Ansätze zur Ko-Evaluation der Daten bzw. Erzählungen mit den Stadtnutzerinnen/nutzern erprobt. Gerade hier würden wir uns einen intensiveren (wissenschaftlichen) Diskurs über bisherige Erfahrungen mit unterschiedlichen Herangehensweisen der KoEvaluation wünschen.

Neben der Einordnung im Diskurs um den Reallaboransatz lassen sich partizipative „Erzählmethoden“ auch als innovatives Instrument der (qualitativen) Datenerhebung mit Anknüpfung an narrative, aber auch partizipative Forschungsansätze der qualitativen Sozialforschung einbetten (Schütze 1983; von Unger 2014). Erzählende Formate sind darüber hinaus auch ohne Kontext des Reallabors für die Quartiersentwicklung spannend - ein Ansatzpunkt, auf den wir in diesem Beitrag nicht weiter eingehen konnten.

Zusammenfassend lässt sich festhalten, dass neue Forschungsinfrastrukturen wie der Reallaboransatz Methoden erfordern, die dem Perspektivwechsel vom Be-Forschen zum Ko-Forschen gerecht werden und den Akteuren erlauben, neue Konzepte ihrer eigenen Rollen zu entwickeln, um Wissen zu erzeugen, das Transformationsprozesse anstoßen kann. Am Beispiel des Forschungsprojekts INTERPART war es unser Ziel darzulegen, dass Formate des Erzählens eine Möglichkeit sein können, um an der Schnittstelle zwischen Wissenschaft und Gesellschaft zu forschen und dadurch Wissen zu erzeugen, das auf den Alltagserfahrungen der Praxisakteure basiert, lokal verortet ist und sich damit auf den konkreten gesellschaftlichen Kontext bezieht. Mit unserem Beitrag wollten wir aufzeigen, dass mit Formaten des Erzählens vielen der Herausforderungen des Reallabors begegnet werden kann.

Förderhinweis Gefördert durch BMBF - Bundesministerium für Bildung und Forschung, Förderkennzeichen 01UM1822AY, Förderprogramm: „Migration und gesellschaftlicher Wandel“ im Rahmen des Forschungsrahmenprogramms „Geistes-, Kultur- und Sozialwissenschaften“ Themenfeld II: Diversität und institutioneller Wandel. 


\section{Literatur}

Altrock, U.; Huning, S.; Peters, D. (2006): Neue Wege in der Planungspraxis und warum aktuelle Planungstheorien unvollständig bleiben. In: Selle, K. (Hrsg.).: Planung neu denken. Band 1: Zur räumlichen Entwicklung beitragen: Konzepte, Theorien, Impulse. Dortmund, 248-263.

Ball-Rokeach, S. J.; Kim, Y.-C.; Matei, S. (2001): Storytelling Neighborhood. Paths to Belonging in Diverse Urban Environments. In: Communication Research 4, 28, 392428. https://doi.org/10.1177/009365001028004003

Bamberg, M.; Georgakopoulou, A. (2008): Small stories as a new perspective in narrative and identity analysis. In: Text \& Talk 28, 3, 377-396. https://doi.org/10.1515/ TEXT.2008.018

Beer, I. (2013): Quartiersentwicklung als Diversitäts- und Teilhabestrategie. Zwischen traditionellen Integrationsdiskursen und gelebten Migrationsrealitäten. In: Schnur, O.; Zakrzewski, P.; Drilling, M. (Hrsg.): Migrationsort Quartier. Zwischen Segregation, Integration und Interkultur. Wiesbaden, 41-53. https://doi.org/10.1007/9783-658-01048-5_3

Björgvinsson, E.; Ehn, P.; Hillgren, P.-A. (2010): Participatory design and "democratizing innovation". In: Robertson, T. (Hrsg.): Proceedings of the 11th Biennial Participatory Design Conference. New York, 41-50. https:// doi.org/10.1145/1900441.1900448

Borner, J.; Kraft, A. H. (2018): Konzeptpapier zur Reallabor-Methode im ENavi-Projekt. http://kmgne.de/ wp-content/uploads/2019/04/ENavi_AP13_KMGNE_ Konzeptpapier_Reallabore_Borner-Kraft_Jan2018.pdf (25.02.2021).

Bruner, J. (2004): Life as Narrative. In: Social Research 71, 3, 691-710.

Dezuanni, M.; Foth, M.; Mallan, K.; Hughes, H.; Osborne, R. (2018): Social Living Labs for Digital Participation and Connected Learning. In: Dezuanni, M.; Foth, M.; Mallan, K.; Hughes, H. (Hrsg.): Digital Participation through Social Living Labs: Valuing Local Knowledge, Enhancing Engagement. Cambridge, 1-17. https:// doi.org/10.1016/C2016-0-01911-3

Eckart, J.; Ley, A.; Häußler, E.; Erl, T. (2018): Leitfragen für die Gestaltung von Partizipationsprozessen in Reallaboren. In: Defila, R.; Di Giulio, A. (Hrsg.): Transdisziplinär und transformativ forschen. Eine Methodensammlung. Wiesbaden, 105-135. https://doi.org/10.1007/9783-658-21530-9_6

Eckstein, B. J.; Throgmorton, J. A. (Hrsg.) (2003): Story and Sustainability. Planning, Practice, and Possibility for American Cities. Cambridge.

Finnegan, R. H. (2004): Tales of the city. A study of narrative and urban life. Cambridge.
Flick, U. (2005): Qualitative Sozialforschung. Eine Einführung. Reinbek bei Hamburg.

Flick, U. (2011): Das Episodische Interview. In: Oelerich, G.; Otto, H.-U. (Hrsg.): Empirische Forschung und Soziale Arbeit. Ein Studienbuch. Wiesbaden, 273-280. https://doi.org/10.1007/978-3-531-92708-4_17

Gerhard, U.; Marquardt, E. (2017): Reallabore als innovatives Forschungsformat zur Untersuchung nachhaltiger Stadtentwicklung - eine kritische Reflexion. In: Berichte. Geographie und Landeskunde 91, 1, 97-111.

Hahne, U. (2019): Reallabore. Katalysator für die nachhaltige Stadt- und Regionalentwicklung? In: RaumPlanung $200,1,50-55$.

Hörning, K.H. (2001): Experten des Alltags. Die Wiederentdeckung des praktischen Wissens. Weilerswist.

Hydén, L.-C. (2013): Bodies, embodiment and stories. In: Andrews, M.; Squire, C.; Tamboukou, M. (Hrsg.): Doing narrative research. London, 126-141.

Joost, G.; Unteidig, A. (2015): Design and Social Change: The Changing Environment of a Discipline in Flux. In: Jonas, W.; Zerwas, S.; von Anshelm, K. (Hrsg.): Transformation Design. Perspectives on a New Design Attitude. Basel, 134-148.

Kanning, H. (2018): Reallabore aus planerischer Perspektive. Hannover. $=$ sustainify Arbeits- und Diskussionspapier 3.

Landolt, S. (2010): Unordentliche Jugendliche an ordentlichen Orten? Raumkonstruktion im Spannungsfeld städtischer Politik, Raumaneignungen Jugendlicher und Bedürfnissen Anwohnender. In: Berichte zur deutschen Landeskunde 84, 3, 237-253.

Lucius-Hoene, G.; Deppermann, A. (2004): Narrative Identität und Positionierung. In: Gesprächsforschung. OnlineZeitschrift zur verbalen Interaktion 5, 1, 166-183.

Mandelbaum, S. J. (1991): Telling Stories. In: Journal of Planning Education and Research 10, 3, 209-214. https:// doi.org/10.1177/0739456X9101000308

Mareis, C. (2010): Entwerfen - Wissen - Produzieren. Designforschung im Anwendungskontext. In: Mareis, C.; Joost, G.; Kimpel, K. (Hrsg.): Entwerfen - Wissen - Produzieren. Designforschung im Anwendungskontext. Bielefeld, 9-32. https://doi.org/10.14361/transcript. 9783839414637.9

Mareis, C. (2011): Design als Wissenskultur. Interferenzen zwischen Design- und Wissensdiskursen seit 1960. Bielefeld.

Martínez, M. (Hrsg.) (2011a): Handbuch Erzählliteratur. Theorie, Analyse, Geschichte. Stuttgart.

Martínez, M. (2011b): Erzählen. In: Martínez, M. (Hrsg.): Handbuch Erzählliteratur. Theorie, Analyse, Geschichte. Stuttgart, 1-11. 
Martínez, M. (Hrsg.) (2017): Erzählen. Ein interdisziplinäres Handbuch. Stuttgart.

Massey, D. (2005): For space. London.

McVee, M. B.; Boyd, F. B. (2015): Exploring diversity through multimodality, narrative, and dialogue: A framework for teacher reflection. New York.

Müller, F. (2018): Designethnografie. Methodologie und Praxisbeispiele. Wiesbaden. https://doi.org/10.1007/ 978-3-658-21388-6

Norrick, N. R. (2010): Conversational Storytelling. In: Herman, D. (Hrsg.): The Cambridge Companion to Narrative. Cambridge, 127-141.

Parodi, O.; Beecroft, R.; Albiez, M.; Quint, A.; Seebacher, A.; Tamm, K.; Waitz, C. (2016): Von „Aktionsforschung“ bis ,Zielkonflikte“. Schlüsselbegriffe der Reallaborforschung. In: Technikfolgenabschätzung in Theorie und Praxis 25, 3, 9-18. https://doi.org/10.14512/tatup. 25.3.9

Polanyi, M. (1985): Implizites Wissen. Frankfurt am Main.

Richter, R.; Rohnstock, N. (2016): Der Erzählsalon als Verfahren strategischen Erzählens. Konzeptionelle Voraussetzungen und empirische Gestalt am Beispiel des Projektes Lausitz an einen Tisch. In: DIEGESIS 5, 2, 84100.

Ricoeur, P. (1984): Time and Narrative. Chicago.

Ricoeur, P. (1986): Life. A Story in Search of a Narrator. In: Doeser, M. C.; Kraay, J. N. (Hrsg.): Facts and Values. Dordrecht, 121-132. https://doi.org/10.1007/97894-009-4454-1

Römhild, R. (2017): Konvivialität - Momente von PostOtherness. In: Hill, M.; Yıldız, E. (Hrsg.): Postmigrantische Visionen. Erfahrungen - Ideen - Reflexionen. Bielefeld, 63-72. https://doi.org/10.14361/9783839439166007

Rössig, W.; Herlo, B.; Moormann, A.; Faber, A.; Diekämper, J. (2018): Visitor Participation. An instrument for enhancing scientific literacy. In: Finlayson, O. E.; McLoughlin, E.; Erduran, S.; Childs, P. (Hrsg.): Electronic Proceedings of the ESERA 2017 Conference. Research, Practice and Collaboration in Science Education. Dublin, 1002-1015.

Rose, M.; Wanner, M.; Hilger, A. (2019): Das Reallabor als Forschungsprozess und -infrastruktur für nachhaltige Entwicklung. Konzepte, Herausforderungen und Empfehlungen. o.O. = NaWiKo Synthese Working Paper 1 .

Sandercock, L. (2003): Out of the Closet: The Importance of Stories and Storytelling in Planning Practice. In: Planning Theory and Practice 4, 1, 11-28. https://doi.org/10. 1080/1464935032000057209

Sandercock, L.; Attili, G. (Hrsg.) (2010): Multimedia Explorations in Urban Policy and Planning. Beyond the
Flatlands. Dordrecht. https://doi.org/10.1007/978-90481-3209-6

Sanders, E. B.-N. (2006): Scaffolds for building everyday creativity. In: Frascara, J. (Hrsg.): Designing Effective Communications: Creating Contexts for Clarity and Meaning. New York, 65-77.

Sanders, E. B.-N. (2013): Perspectives on Participation in Design. In: Mareis, C.; Held, M.; Joost, G. (Hrsg.): Wer gestaltet die Gestaltung? Praxis, Theorie und Geschichte des partizipatorischen Designs. Bielefeld, 65-78. https:// doi.org/10.14361/transcript.9783839420386.65

Schäpke, N.; Stelzer, F.; Bergmann, M.; Singer-Brodowski, M.; Wanner, M.; Caniglia, G.; Lang, D. J. (2017): Reallabore im Kontext transformativer Forschung. Ansatzpunkte zur Konzeption und Einbettung in den internationalen Forschungsstand. Lüneburg. = IETSR Discussion Papers in Transdisciplinary Sustainability Research 1/2017.

Schneidewind, U. (2014): Urbane Reallabore. Ein Blick in die aktuelle Forschungswerkstatt. In: pnd|online 3, 1-7.

Schütze, F. (1983): Biographieforschung und narratives Interview. In: Neue Praxis 13, 3, 283-293.

SEG - Stadtentwicklungsgesellschaft Wiesbaden (2020): Integriertes Städtebauliches Entwicklungskonzept: ,Soziale Stadt' Biebrich-Mitte. Wiesbaden.

Solorzano, D. G.; Yosso, T. J. (2001): Critical race and LatCrit theory and method: Counter-storytelling. In: International Journal of Qualitative Studies in Education 14, 4, 471-495. https://doi.org/10.1080/09518390110063365

Sommer, R. (2017): Gruppenbildung. In: Martínez, M. (Hrsg.): Erzählen. Ein interdisziplinäres Handbuch. Stuttgart, 257-259.

Strauss, A. L.; Corbin, J.M. (1997): Grounded theory in practice. Thousand Oaks.

Throgmorton, J. A. (1992): Planning as Persuasive Storytelling About the Future. Negotiating an Electric Power Rate Settlement in Illinois. In: Journal of Planning Education and Research 12, 1, 17-31. https://doi.org/10.1177/ 0739456 X9201200103

Throgmorton, J. A. (2003): Planning as Persuasive Storytelling in a Global-Scale Web of Relationships. In: Planning Theory 2, 2, 125-151. https://doi.org/10.1177/ 14730952030022003

Unteidig, A.; Domínguez Cobreros, B.; Calderon-Lüning, E.; Joost, G. (2017): Digital commons, urban struggles and the role of Design. In: The Design Journal 20, S1, 3106-3120. https://doi.org/10.1080/14606925.2017. 1352818

Vacchelli, E. (2018): Embodied research in migration studies. Using creative and participatory approaches. Bristol.

van Hulst, M. (2012): Storytelling, a model of and a model 
for planning. In: Planning Theory 11, 3, 299-318. https:// doi.org/10.1177/1473095212440425

von Unger, H. (2014): Partizipative Forschung. Einführung

in die Forschungspraxis. Wiesbaden. https://doi.org/10.

1007/978-3-658-01290-8 